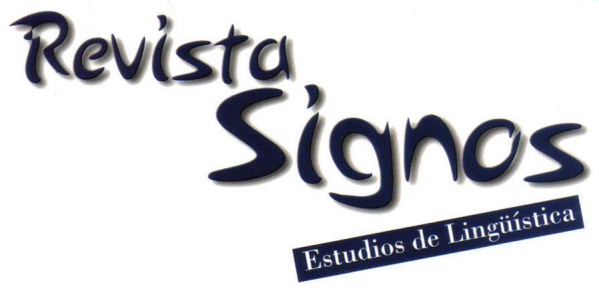

\title{
Diferencias de género en evaluaciones de narraciones de experiencias personales en el habla juvenil de Santiago de Chile. Una aproximación sociolingüística
}

\author{
Gender differences in evaluations of personal narrations in the speech \\ of young people from Santiago, Chile. A sociolinguistic approach
}

\author{
Silvana Guerrero \\ siguerrero@u.uchile.cl \\ Universidad de Chile \\ Chile
}

Recibido: 27-IV-2010 / Aceptado: I5-X-2010

Resumen: En esta investigación se pretende dar cuenta de las diferencias de género en el componente evaluativo (Labov \& Waletzky, 1967; Labov, 1972) en un sub-tipo específico del discurso narrativo juvenil en Santiago de Chile, a saber, en las narraciones de experiencias personales. Dichas diferencias fueron relevadas en una muestra de 64 entrevistas sociolingüísticas ( 32 masculinas y 32 femeninas) estratificadas socioculturalmente, conformando, de esta forma, una muestra significativa de discurso natural grabado en el estilo que Labov (1972) denomina como 'vernacular'. La hipótesis que sustenta la investigación es la existencia de diferencias de género en el discurso narrativo juvenil, específicamente, en lo concerniente al componente evaluativo; por lo tanto, el objetivo principal de la indagación consistió en recopilar las mencionadas diferencias y establecer su incidencia en el desarrollo de las narraciones, en el caso de que existieran. Tras el análisis de los 64 relatos, se verificó la existencia de diferencias de género en el mencionado constituyente de las narraciones, presente en 61 de las 64 historias. Nuestro trabajo incluyó, también, un análisis de siete categorías evaluativas (emoción, percepción, cognición, estado físico, intención, relación y habla reportada), según la propuesta de Jiménez (2006), donde se concluyó que, en la mayoría de las ocasiones, son las mujeres las que emplean la mayor cantidad de elementos de evaluación. Por último, podrían explicarse las diferencias de género relevadas recurriendo a la relativa mayor conciencia que las mujeres tienen de la importancia de la cultura oral.

Palabras Clave: Narraciones de experiencias personales, habla juvenil, componente evaluativo, categorías evaluativas. 


\begin{abstract}
This study presents gender differences in the evaluative component (Labov \& Waletzky, 1967; Labov, 1972) in a specific sub-type of narrative discourse in young people from Santiago, Chile: personal narratives. These differences were collected from a sample of 64 (32 female; 32 males) culturally stratified sociolinguistic interviews. Thus, a representative sample of recorded natural discourse was comprised, what Labov (1972) calls vernacular. The hypothesis is the existence of gender differences in the narrative discourse of young speakers, especially in relation to the evaluative component; therefore, the main objective of this investigation consisted in establishing the occurrence of these differences, if any, in the development of the narrations.After analyzing all 64 accounts, the existence of gender differences in the evaluative constituent of narrations was detected in 6l of them. Following Jiménez (2006), this study also includes an analysis of seven evaluative categories (emotion, perception, cognition, physical state, intention, relation, and reported speech), whereby it was established that it is women who mostly use evaluative elements. Finally, the gender differences collected for this study could be explained by women's relatively deeper awareness of the importance of orality.
\end{abstract}

Key Words: Personal narratives, speech of the youth, evaluative component, evaluative categories.

\section{INTRODUCCIÓN}

En esta investigación se analizarán las diferencias de género en el componente evaluativo en un sub-tipo específico del discurso narrativo juvenil en Santiago de Chile, a saber, en las narraciones de experiencias personales. Nuestro trabajo se enmarca, entonces, en el amplio dominio del análisis sociolingüístico del discurso. El interés por el análisis del discurso oral se ha acrecentado de manera notable en los últimos años (De Beaugrande, 2000; van Dijk, 2005; Charaudeau \& Maingueneau, 2005). Por lo que respecta a la relación existente entre género y discurso, esta encuentra un sostenido y creciente desarrollo a partir de la década de 1960. De esta forma, se ha generado un sinnúmero de trabajos abocados a indagar dicha relación, sobre todo, en el contexto de la lingüística norteamericana (Lakoff, 1973; Jones, 1980; Tannen, 1990a, 1990b, 1996; Coates, 2009), situación que no ha alcanzado un nivel comparable en el ámbito hispánico.

Por ello, nuestro análisis intenta ser un aporte a esta área de la investigación sociolingüística aplicada a la lengua española. En consideración a lo anteriormente expuesto, este estudio tiene carácter exploratoriodescriptivo aplicado a una muestra de entrevistas sociolingüísticas. Para nuestra investigación, hemos seleccionado el habla del segmento etario juvenil de la población, ya que la investigación sobre este grupo de edad ha mostrado que se trata del sector más proclive al empleo de formas innovadoras (Blas Arroyo, 2005). En este estudio se sustenta la hipótesis de la existencia de diferencias de género en las evaluaciones del discurso narrativo juvenil de mujeres y hombres. Por consiguiente, nuestra indagación deberá, por un lado, verificar si existen o no tales diferencias $y$, por otro, analizar aquellas que se encuentren, a la luz de las categorizaciones pragmático-discursivas que las justifiquen de modo más adecuado.

\section{Marco conceptual}

\section{I.I. El discurso narrativo y la propuesta de Labov y Waletzky (1967)}

A partir de la concepción de los componentes (o niveles) del texto de Werlich (1975) y del concepto de superestructura de van Dijk (2000), Adam (1999) plantea secuencias discursivas prototípicas, que responden a tipos relativamente estables de combinación de enunciados, organizados mediante su estructura jerárquica interna (esquema) y por su unidad compositiva (plan). El autor señala que se trata de unidades modélicas, sintetizadas en cinco tipos de secuencias, como sigue: a) narrativa, b) descriptiva, c) argumentativa, d) explicativa $y, f$ ) dialogal. De acuerdo con Bassols y Torrent (2003), la narración es el tipo de secuencia discursiva que ha generado el mayor número de estudios, análisis y reflexiones. Las narraciones de experiencias personales constituyen una de las formas de expresión verbal más utilizadas por las personas. Por otra parte, según Calsamiglia y Tusón (1999), no solo se narra para contar historias, sino que las narraciones están presentes también en los diálogos, en las anécdotas, en los discursos argumentativos y 
en los chistes, entre otras instancias específicas de uso lingüístico. Asimismo, en concordancia con lo señalado por Moreno Fernández (1998), la narración oral es una de las estructuras (o subestructuras) textuales más interesantes y complejas que pueden incorporarse dentro de las conversaciones.

La bibliografía sobre narraciones es extensa; sin embargo, el trabajo seminal de Labov y Waletzky (1967), así como el de Labov (1972), han demostrado ser por mucho el modelo de más alto rendimiento empírico en la investigación sobre el problema. En estricto sentido, Labov (1972: 360) define la narración como:

"one method of recapitulating past experience by matching a verbal sequence of clauses to the sequence of events which (it is inferred) actually occurred [un método de recapitulación de la experiencia pasada adecuando una secuencia verbal de proposiciones a la secuencia de sucesos que (se supone) ocurrieron realmente (la traducción es nuestra)]".

De esta forma, la narración es considerada como un texto que expresa una serie de eventos que ocurren en el tiempo, pero donde, a su vez, se percibe de manera subyacente una relación causal o temática. Labov y Waletzky (1967) y Labov (1972), distinguen dos tipos de relatos: los 'relatos mínimos', compuestos de -al menos- dos cláusulas narrativas en pasado y los 'relatos completos'. Dichos autores plantean que una narración de experiencias personales plenamente formada exhibe seis rasgos estructurales bien definidos, cada uno con una posición característica en la narración. A continuación se describen los seis rasgos estructurales de una secuencia narrativa plenamente constituida:

I) Resumen: este rasgo no aparece en el artículo de 1967, sino que es posterior. Las narraciones se introducen, aunque no siempre, a través de 'que', generalmente, consiste en una proposición general que la narración va a ejemplificar.

2) Orientación: una narración prototípica comienza haciendo referencia a un tiempo, un lugar, unas personas y una conducta esperada en cierta situación. Esos son los componentes de una orientación narrativa.

3) Acción de complicación de la narración: corresponde al núcleo o esqueleto de la narración en el que aparecen los distintos eventos narrados, constituyendo, de esta forma, el clímax del relato.
4) Evaluación: esta sección es utilizada por el narrador para validar su narración, es decir, para dejar en claro cuál es la razón de ser de su relato y su meta al narrarlo. De este modo, la evaluación marca la parte central o informativa de un relato, aunque puede ser transversal a este. La evaluación no constituye estrictamente una parte, sino está conformada por todos los fragmentos en que el narrador utiliza medios que hacen de la historia un relato interesante.

5) Resultado o resolución: la resolución dice lo que ocurrió finalmente, es decir, corresponde al término del relato de una serie de eventos, por lo tanto, está marcado, la mayoría de las veces, por cláusulas narrativas; de hecho, en el desenlace aparece la última de ellas.

6) Coda: se trata de la moraleja. Generalmente, una cláusula narrativa lleva implícita una pregunta 'y entonces, ¿qué ocurrió?'. Cuando las preguntas que van surgiendo quedan respondidas, puede aparecer la coda, con la que el narrador regresa al presente, haciéndole saber a su oyente que la narración ha concluido (adaptado de Labov \&Waletzky, 1967).

Cabe señalar que para los fines de nuestra investigación, solo se analizará el componente evaluativo, dado que, en general, se manifiesta de manera transversal en los relatos, a fin de justificarlos adecuadamente; en este sentido, la evaluación constituye un punto clave dentro de la historia. Asimismo, es el componente evaluativo el que dota a la narración de mayor dinamismo, haciéndola más amena y entretenida para los oyentes (Labov \& Waletzky, 1967; Labov, 1972). En la sección siguiente, se ampliará la evaluación laboviana a partir de la propuesta de Jiménez (2006).

\section{I.I.I. Las categorías evaluativas}

De acuerdo con Jiménez (2006), es importante recordar que los seres humanos reaccionan internamente, tienen emociones y motivaciones, y las narraciones responden a dichas emociones internas, porque permiten expresar el punto de vista de quien narra. A esta percepción o punto de vista de los hechos es a lo que se le denomina 'evaluación', que de acuerdo con Labov y Waletzky (I967), es el elemento clave de una narración. Para Shiro (1998), aquellas categorías que hacen referencia a las emociones, intenciones o percepciones, reflejan tipos de evaluaciones a través de los cuales se alude a la posición del personaje en el mundo narrado, 
por lo tanto, son la expresión de un propósito o de una meta. A partir de estos supuestos, Jiménez propone siete categorías evaluativas que explicamos a continuación:

I) Emoción (EMO): expresa afecto o emoción, por ejemplo, 'se puso contenta'.

2) Cognición (COG): representa el pensamiento y las creencias, por ejemplo, ' el pensó que era un pajarito'.

3) Percepción (PER): expresa todo lo que se percibe a través de los sentidos, por ejemplo, 'vio al policía'.

4) Estado físico (PHY): se refiere al estado interno más físico que emocional, por ejemplo, 'estaba muy cansada'.

5) Intención (INT): expresa las intenciones de un personaje al realizar alguna acción, por ejemplo, 'trató de escapar'.

6) Relación (REL): representa una acción que enfatiza la interpretación de una relación entre personajes o entre un personaje y un objeto, más que la acción en sí misma, por ejemplo, 'encontraron al ratoncito'.

7) Habla reportada (RPS): reporta el lenguaje que cita al hablar. Se manifiesta a través de tres subtipos:

a. Habla reportada directa (RPD): las.palabras del personaje se reportan como si el personaje las hubiera pronunciado textualmente, por ejemplo, 'le dijo: por aquí, señor, por favor'.

b. Habla reportada indirecta (RPI): las palabras del personaje son reportadas indirectamente, por ejemplo, 'mi mamá le dijo que yo estaba ahi'.

c. Habla reportada libre (RPF): las expresiones léxicas significan que hubo un intercambio verbal sin reportar explícitamente las palabras pronunciadas, pero reportando frecuentemente el acto de habla realizado, es decir, el propósito comunicativo, por ejemplo, “mi mamá me regañó”.

\section{I.2. Las variables sociolingüísticas y los factores sociales}

El interés de la sociolingüística por el estudio de las variables lingüísticas es fundamental, ya que el desarrollo de esta interdisciplina se basa en que gran parte de los hechos de variación lingüística no varían de manera azarosa, sino que de forma sistemática si se los correlaciona con variables demosociales como la clase social, la edad y el sexo-género. Esto implica que el hecho de que las variables lingüísticas estén en covariación sistemática con otras variables permite a los individuos situarse en un determinado espacio del espectro social. A este respecto, Coates (2009: 89) señala que:

"El uso de las variables lingüísticas es una de las formas en que los hablantes pueden ubicarse en el espacio social. Es decir, aunque desde el punto de vista lingüístico las variables lingüísticas sean formas equivalentes para referirse a algo en particular, son diferentes desde una perspectiva social".

Según Moreno Fernández (1998), la investigación sociolingüística ha permitido determinar que las variables sociales que influyen sobre la variación lingüística lo hacen de una forma específica en cada comunidad de habla y respecto de fenómenos lingüísticos concretos. Por su parte, Blas Arroyo (2005) señala que no es posible reconocer de antemano qué tipo de variables sociales van a actuar sobre la variación lingüística, debido a que la variabilidad no tiene por qué manifestarse de la misma forma en comunidades de habla diferentes y, además, porque estos mismos factores no se configuran de la misma forma en sociedades distintas. Así ocurre con las variables 'sexo', 'edad' y 'clase social'. Como ya lo señalamos, el factor sexo, en primer lugar, y la clase social, en segundo término, serán las variables independientes empleadas en nuestra investigación sobre las diferencias de género en narraciones de hablantes jóvenes de Santiago de Chile.

En relación con el factor sexo, consideramos necesario precisar que dicha variable se ha considerado, tradicionalmente, según López Morales (2004), por un lado, en lo referido al sexo como categoría biológica y, por otro, en lo que respecta al género en tanto construcción sociocultural. En este sentido, Cheshire (2003) señala que el término 'sexo' ha sido empleado para referirse a la distinción fisiológica entre mujeres y hombres, mientras que 'género' lo ha sido para la elaboración social y cultural de sexos diferentes, en un proceso vinculado con los roles sociales, las oportunidades y las expectativas. Sin embargo, de acuerdo con esta autora, puesto que el proceso comienza con el nacimiento, podría discutirse si 'género' es el término más apropiado para esta categoría antes que 'sexo'. No obstante, ambos términos se encuentran en la literatura del variacionismo usados 
a veces en una manera, al parecer, indistinta pero, en otras ocasiones, empleados para distinguir entre las características biológicas y los factores sociales de los sujetos.

\section{I.3. Los estudios de género y discurso}

Según Moreno Fernández (1998), la mayor parte de lo que hoy sabemos acerca de la conducta lingüística de hombres y mujeres se lo debemos a la sociolingüística. Los estudios sobre la relación entre género y discurso surgen en la década de los setenta, específicamente, en 1975. Siguiendo a Prieto y San Martín (2002-2003), estos primeros trabajos eran un intento por caracterizar el habla de mujeres y hombres, pero sin trascender al ámbito de la explicación de estas características. En etapas siguientes, en cambio, los enfoques son más bien explicativos. De esta forma, hoy destacan investigaciones como las de Tannen (1990a), quien enfatiza el carácter intercultural de la interacción conversacional al plantear la tesis de que los hombres y las mujeres utilizan estilos conversacionales diferentes. En este sentido, el interés del hombre radicaría en comunicar información y mantener la independencia y el estatus frente a su interlocutor, mientras que las mujeres estarían más preocupadas por establecer y mantener una relación a nivel interpersonal. En atención a este principio, es importante precisar que en esta investigación será central el enfoque de las diferencias culturales, puesto que la idea que subyace a las diferencias de género en el discurso narrativo juvenil es que, tal como lo precisan estudios como los de Tannen (1990b), los hombres y las mujeres poseen estilos conversacionales distintos no por azar, sino porque sus procesos de socialización son, asimismo, diferentes.

La incidencia de la variable de género en el discurso es un problema que ha sido abordado de un modo especial en la lingüística norteamericana, entre otros, por Lakoff (1973), Coates (1987) y Tannen (1990a). De gran utilidad para los propósitos de esta investigación, resultó ser el panorama del desarrollo histórico de los estudios sobre género y discurso que presentan Prieto y San Martín (20022003). Según estos últimos autores, los factores que explicarían los rasgos atribuidos al habla femenina en la investigación empírica, podrían explicarse a través de la propuesta de Coates basada en una apreciación del trabajo de Jones (1980) sobre el cotilleo femenino. Estos factores serían: I) la situación comunicativa, 2) los participantes, 3) el tópico y 4) las funciones
- metas del acto comunicativo. Otro aspecto importante en relación con los estudios sobre el discurso narrativo y las diferencias de género, es el que se vincula con el discurso referido y la oralidad. En su investigación sobre, diferencias de género en el empleo del discurso referido, los autores citados concluyen que existe un uso preponderante de estilo directo en las interacciones femeninas, lo que podría explicarse por una reminiscencia de la 'oralidad primaria' en una sociedad que ha devenido en una cultura preponderantemente escrita'; es decir, entre los representantes del género masculino se habría perdido este rasgo de oralidad como consecuencia del paso del orden tradicional a la modernidad, mientras que entre las representantes del género femenino dicho rasgo pervive con mayor frecuencia al haber accedido las mujeres a la educación formal y a la comunicación escrita con bastante posterioridad a los varones.

A partir de los aportes teóricos de los autores antes señalados, nuestro trabajo se concentrará en el análisis de las diferencias de género del componente evaluativo en un sub-tipo específico del discurso narrativo juvenil en Santiago de Chile: las narraciones de experiencias personales.

\section{Marco metodológico}

\section{I. Corpus}

La muestra que sirve de base para nuestro estudio corresponde a 64 entrevistas sociolingüísticas realizadas en la cátedra de Sociolingüística, a cargo del profesor Dr. Luis Prieto Vera, de las Licenciaturas en Lengua y Literatura Hispánica y Lengua y Literatura Inglesas de la Universidad de Chile. Las entrevistas fueron hechas a hombres y mujeres jóvenes con características sociodemográficas congruentes $y$, aplicando los supuestos metodológicos sugeridos por Labov (1992), en dichas situaciones los entrevistadores debían tratar de superar la 'paradoja del observador' 2 consiguiendo, de esta forma, una muestra significativa de discurso natural grabado (estilo 'vernacular') de hablantes representativos de la comunidad de habla en estudio. El cuestionario utilizado para la realización de las mencionadas entrevistas consta de una sección cuya función es elicitar las narraciones de experiencias personales que conforman el corpus de nuestra investigación.

De cada una de dichas entrevistas se extrajo el segmento narrativo mejor desarrollado por los entrevistados. En concreto, se trata de 64 entrevistas 
grabadas, realizadas a jóvenes santiaguinos de entre 20 y 34 años, estratificados por sexo y nivel socioeconómico, según la propuesta de Prieto (I 995 1996). Las entrevistas que conforman la muestra del estudio fueron grabadas entre 2005 y 2009 , y una parte del mencionado material, específicamente 36 entrevistas, forma parte del Proyecto para el Estudio Sociolingüístico del Español de España y América (PRESEEA), específicamente, del corpus de PRESEEA Santiago de Chile. El resto de las entrevistas seleccionadas pertenecen al corpus del proyecto de Estudio Sociolingüístico del Español de Chile $(\mathrm{ESECH})^{3}$.

\section{I.I. Procedimiento de estratificación social utilizado}

De acuerdo con Prieto (1995-1996), la muestra del estudio se estratificó convenientemente, utilizando una escala de adscripción de estatus socioeconómico, basada en la combinación de cuatro variables: I) nivel educacional, 2) profesión u ocupación, 3) comuna de residencia $y, 4)$ ingreso familiar mensual. Dicha escala incluye cuatro estratos socioeconómicos, a saber, bajo, medio bajo, medio y medio alto.

\section{I.2. Población y muestra}

En nuestra investigación se consideró la población constituida por hombres y mujeres de la Región Metropolitana, de entre 20 y 34 años de edad, que corresponden al segmento etario juvenil de la población santiaguina. Al respecto, los criterios de asignación de hablante nativo de Santiago de Chile son los siguientes: I) haber nacido y residido en forma ininterrumpida en Santiago; 2) haber residido en forma ininterrumpida en Santiago desde los cinco años de edad y; 3 ) haber nacido en Santiago y haber residido en Santiago la mayor parte de sus vidas, salvo por periodos que sumados no superen los cuatro años en el tramo de 50 años y más y los tres años en el tramo de 35 a 49 años.

El cuestionario se aplicó a una muestra del tipo denominado 'muestra por cuotas', en la que se divide a la población en estratos o categorías y se asigna una cuota a cada uno de los distintos estratos. La muestra así conformada comprende un total de 64 individuos, distribuidos proporcionalmente como se indica en la Tabla I que se incluye a continuación.
Tabla I. Distribución de sujetos de la muestra por nivel socioeconómico, sexo y edad según ESECH.

\begin{tabular}{|l|c|c|c|}
\hline \multirow{4}{|c|}{ Grupo de edad } \\
\hline \multirow{2}{*}{$\begin{array}{l}\text { Nivel } \\
\text { socioeconómico }\end{array}$} & \multicolumn{2}{|c|}{$20-34$} & Totales \\
\cline { 2 - 4 } & $\mathrm{H}$ & $\mathrm{M}$ & \\
\hline Medio alto & 8 & 8 & 16 \\
\hline Medio & 8 & 8 & 16 \\
\hline Medio bajo & 8 & 8 & 16 \\
\hline Bajo & 8 & 8 & 16 \\
\hline Totales & 32 & 32 & 64 \\
\hline
\end{tabular}

En la Tabla 2, por su parte, se presentan las características sociodemográficas de cada uno de los integrantes de la muestra, clasificados de acuerdo con su estrato socioeconómico y su sexo-género.

\section{Presentación y análisis de los resultados}

\section{I. Análisis del componente evaluativo en las narraciones}

Para el análisis del componente evaluativo de las narraciones que conforman nuestro corpus, nos basaremos, básicamente, en los planteamientos de Labov y Waletzky (1967) y Labov (1972). Lo que pretendemos en este apartado es dar cuenta de la posible existencia de diferencias de género en dicho constituyente, abarcando, además, el factor clase social. La evaluación constituye un punto clave en el relato, porque en ella el narrador da cuenta de la razón por la que narra su historia. No obstante, quien relata podría prescindir, fácilmente, de este componente narrativo, al igual que del resumen o de la coda. En general, las evaluaciones son transversales al relato y no ocupan un lugar específico dentro de él. Incluso, el narrador suele reiterarla en varias ocasiones para validar aún más su relato. Siguiendo las teorías de Labov y Waletzky (1967) y Labov (1972), se analizarán formalmente las evaluaciones presentes en las narraciones juveniles de nuestro corpus en búsqueda de diferencias de género, pero, además, se examinará este componente narrativo desde el punto de vista de las categorías evaluativas, siguiendo la propuesta de Jiménez (2006). Antes de exponer el análisis detallado del componente evaluativo registrado en el corpus, presentamos el Gráfico I, que da cuenta de las diferencias de género observadas respecto de este constituyente en las narraciones que conforman nuestra investigación. 
Tabla 2. Características sociodemográficas de los integrantes de la muestra.

\begin{tabular}{|c|c|c|c|c|c|c|c|}
\hline $\begin{array}{l}\text { Grupo } \\
\text { de edad }\end{array}$ & Estrato & Sexo & Código & Edad & $\begin{array}{c}\text { Nivel } \\
\text { educacional }\end{array}$ & Profesión u ocupación & $\begin{array}{l}\text { Comuna de } \\
\text { residencia }\end{array}$ \\
\hline \multirow{64}{*}{ 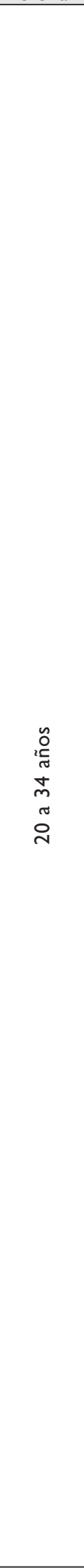 } & \multirow{16}{*}{ 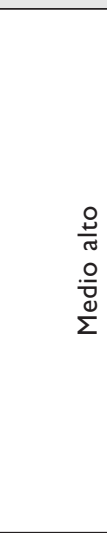 } & \multirow{8}{*}{ 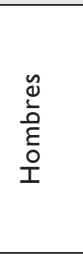 } & $\mathrm{HI} 8 \mathrm{I}^{4}$ & 23 & $U \mathrm{UI}^{5}$ & Estudiante de Diseño Industrial & Providencia \\
\hline & & & $\mathrm{HI} 82$ & 24 & UC & Licenciado en Derecho & Peñalolén \\
\hline & & & $\mathrm{HI} 83$ & 26 & $\mathrm{UI}$ & Operador de Sistemas Informáticos & Peñalolén \\
\hline & & & $\mathrm{HI} 84$ & 25 & UI & Estudiante de Literatura & Vitacura \\
\hline & & & $\mathrm{HI} 85$ & 22 & UI & Estudiante de Filosofía & Ñuñoa \\
\hline & & & $\mathrm{HI} 86$ & 25 & $\mathrm{UI}$ & Estudiante de Ingeniería Industrial & Peñalolén \\
\hline & & & $\mathrm{HI} 87$ & 26 & UC & Constructor Civil & Providencia \\
\hline & & & $\mathrm{HI} 88$ & 21 & $\mathrm{UI}$ & Estudiante de Historia & Ñuñoa \\
\hline & & \multirow{8}{*}{$\frac{\stackrel{y}{0}}{\frac{d}{3}}$} & MI9I & 31 & UC & Psicóloga & Ñuñoa \\
\hline & & & MI92 & 21 & $\mathrm{UI}$ & Estudiante de Medicina & Las Condes \\
\hline & & & MI93 & 25 & UC & Licenciada en Inglés & Ñuñoa \\
\hline & & & MI94 & 21 & $\mathrm{UI}$ & Estudiante de Terapia Ocupacional & Ñuñoa \\
\hline & & & MI95 & 28 & UC & Médico psiquiatra & Providencia \\
\hline & & & MI96 & 21 & UI & Estudiante de Teatro & Ñuñoa \\
\hline & & & MI97 & 23 & $\mathrm{UI}$ & Estudiante de Filosofía & Las Condes \\
\hline & & & MI98 & 26 & $\mathrm{UI}$ & $\begin{array}{l}\begin{array}{l}\text { Estudiante de Licenciatura en } \\
\text { Criminalística }\end{array} \\
\end{array}$ & Peñalolén \\
\hline & \multirow{16}{*}{$\frac{\frac{0}{0}}{\sum^{\frac{0}{2}}}$} & \multirow{8}{*}{$\begin{array}{l}\tilde{y} \\
\frac{0}{\varepsilon} \\
\text { है } \\
\text { I }\end{array}$} & $\mathrm{HI} 2 \mathrm{I}$ & 23 & $\mathrm{UI}$ & Estudiante de Física & La Florida \\
\hline & & & $\mathrm{HI22}$ & 26 & UC & Profesor de Educación Física & Maipú \\
\hline & & & $\mathrm{HI} 23$ & 22 & $\mathrm{UI}$ & $\begin{array}{llll}\begin{array}{l}\text { Estudiante } \\
\text { Castellano }\end{array} & \text { de } & \text { Pedagogía } & \text { en } \\
\end{array}$ & Ñuñoa \\
\hline & & & $\mathrm{HI} 24$ & 25 & UI & Estudiante de Inglés & La Florida \\
\hline & & & $\mathrm{HI} 25$ & 22 & TPI & Estudiante de Geografía & Santiago \\
\hline & & & $\mathrm{HI} 26$ & 25 & UI & Estudiante de Arquitectura & Santiago \\
\hline & & & $\mathrm{HI} 27$ & 22 & UI & Estudiante de Diseño Gráfico & Macul \\
\hline & & & $\mathrm{HI} 28$ & 21 & UI & Estudiante de Arquitectura & Maipú \\
\hline & & \multirow{8}{*}{$\begin{array}{l}\frac{y}{\frac{1}{0}} \\
\frac{d}{2}\end{array}$} & MI3I & 24 & TPC & Técnico paramédico & Santiago \\
\hline & & & MI32 & 30 & TPC & Administradora de Local Comercial & Las Condes \\
\hline & & & MI33 & 25 & UC & Profesora de Inglés & Maipú \\
\hline & & & MI34 & 25 & UC & Parvularia & Las Condes \\
\hline & & & MI35 & 28 & UI & Estudiante de Inglés & Santiago \\
\hline & & & MI36 & 21 & UI & Estudiante de Fonoaudiología & Santiago \\
\hline & & & MI37 & 26 & UI & Estudiante de Enfermería & Ñuñoa \\
\hline & & & MI38 & 23 & UI & Estudiante de Medicina Veterinaria & Puente Alto \\
\hline & \multirow{16}{*}{$\begin{array}{l}\frac{0}{\pi} \\
\text { J } \\
.0 \\
\frac{0}{\sigma} \\
\Sigma\end{array}$} & \multirow{8}{*}{$\begin{array}{l}\tilde{0} \\
\frac{1}{\tilde{\varepsilon}} \\
\text { 운 }\end{array}$} & $\mathrm{H} 06 \mathrm{I}$ & 29 & MC & Chofer de colectivo & Puente Alto \\
\hline & & & $\mathrm{H} 062$ & 32 & MC & Operario de bodega & Maipú \\
\hline & & & $\mathrm{H} 063$ & 20 & MC & Reponedor de supermercado & La Florida \\
\hline & & & $\mathrm{H} 064$ & 32 & MC & Transportista & San Bernardo \\
\hline & & & $\mathrm{H} 065$ & 25 & TPC & Fotocopiador & Estación Central \\
\hline & & & $\mathrm{H} 066$ & 30 & TPI & Estudiante de Informática & Puente Alto \\
\hline & & & $\mathrm{H} 067$ & 25 & MC & Vendedor & Puente Alto \\
\hline & & & $\mathrm{H} 068$ & 20 & UIC & Cesante & La Florida \\
\hline & & \multirow{8}{*}{ 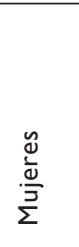 } & M07I & 32 & MC & Lavandera & La Cisterna \\
\hline & & & M072 & 28 & TPC & Esteticista & La Florida \\
\hline & & & M973 & 24 & MTPC & Ayudante de cocina & San Ramón \\
\hline & & & M074 & 20 & TPC & Cesante & Quinta Normal \\
\hline & & & M075 & 32 & MC & Vendedora & Puente Alto \\
\hline & & & M076 & 28 & MC & Contadora & Quinta Normal \\
\hline & & & M077 & 20 & MC & Promotora & Puente Alto \\
\hline & & & M078 & 22 & TPI & Estudiante Técnico en Enfermería & Puente Alto \\
\hline & \multirow{16}{*}{$\frac{\circ}{\pi}$} & & $\mathrm{HOOI}$ & 21 & MI & Obrero de construcción & San Bernardo \\
\hline & & & $\mathrm{H} 002$ & 28 & MI & Despachador de guías & Independencia \\
\hline & & & $\mathrm{H} 003$ & 27 & MI & Cesante & El Bosque \\
\hline & & قِ & $\mathrm{H} 004$ & 25 & MTPI & Reparador de computadores & Maipú \\
\hline & & $\varepsilon$ & $\mathrm{H} 005$ & 21 & MI & Reponedor de supermercado & Puente Alto \\
\hline & & I & $\mathrm{H} 006$ & 34 & MI & Chofer & Huechuraba \\
\hline & & & $\mathrm{H} 007$ & 25 & $\mathrm{BI}$ & Guardia de seguridad & La Pintana \\
\hline & & & $\mathrm{H} 008$ & 20 & MI & Estudiante de enseñanza media & San Ramón \\
\hline & & & MOII & 27 & MI & Vendedora de tienda & El Bosque \\
\hline & & & MOI2 & 32 & $\mathrm{BI}$ & Cesante & San Bernardo \\
\hline & & & MOI3 & 29 & MI & Dueña de casa & El Bosque \\
\hline & & $\frac{0}{0}$ & Mol4 & 33 & MI & Dueña de casa & Quinta Normal \\
\hline & & $\frac{3}{3}$ & M0I5 & 23 & $B C$ & Dueña de casa & Maipú \\
\hline & & $\Sigma$ & M0I6 & 20 & $\mathrm{MI}$ & Cesante & La Granja \\
\hline & & & M0I7 & 29 & MI & Asesora del hogar & San Ramón \\
\hline & & & M0I8 & 31 & MI & Promotora & Lo Espejo \\
\hline
\end{tabular}


Gráfico I. Gráfico general de frecuencia de evaluaciones registrada en el corpus.

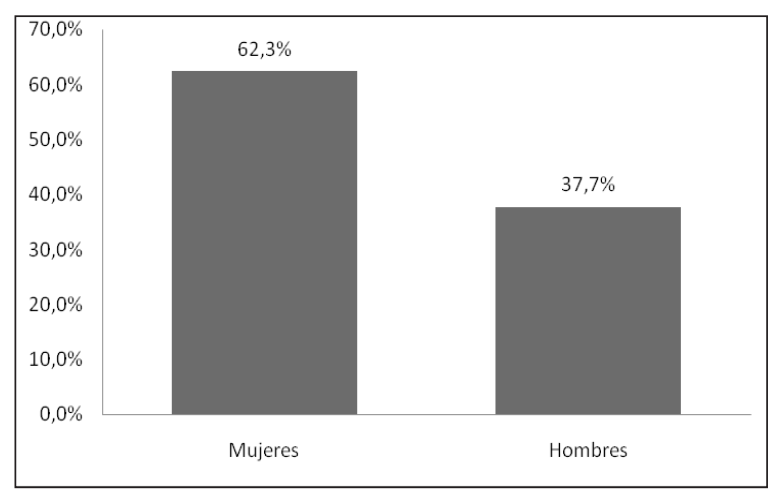

De las 64 narraciones que conforman nuestra muestra, $6 \mathrm{I}$ incluyen el componente evaluativo, con una frecuencia variable de elementos evaluativos cada una, que sumados corresponden a 268 casos. Como puede observarse en el Gráfico I, de este total, el $62,3 \%$ (167/268) de las ocasiones en que se registra este componente corresponde a mujeres, en contraste con el $37,7 \%$ ( $10 \mathrm{I} / 268)$ que es utilizado por hombres. Cabe destacar, asimismo, que la evaluación

Tabla 3. Tabla general de frecuencia de evaluaciones registrada en el corpus.

\begin{tabular}{|l|c|c|}
\hline \multicolumn{1}{|c|}{ Estrato } & Sexo & $\begin{array}{c}\text { Total } \\
\text { evaluaciones }\end{array}$ \\
\hline Medio alto & Hombres & 21 \\
\cline { 2 - 3 } & Mujeres & 55 \\
\hline Medio & Hombres & 18 \\
\cline { 2 - 3 } & Mujeres & 41 \\
\hline \multirow{2}{*}{ Medio bajo } & Hombres & 30 \\
\cline { 2 - 3 } & Mujeres & 31 \\
\hline \multirow{2}{*}{ Bajo } & Hombres & 32 \\
\cline { 2 - 3 } & Mujeres & 40 \\
\hline TOTAL & & 268 \\
\hline
\end{tabular}

es el constituyente de la estructura narrativa que más se emplea en las narraciones, pues del total de relatos que conforma nuestro corpus, solo no se registra en tres ocasiones, correspondientes a relatos masculinos. Esta situación puede explicarse, en primera instancia, por la necesidad del narrador de señalar explícitamente el motivo por el que está contando su historia $y$, en segundo lugar, para entregar al oyente un discurso mucho más 'entretenido', 'dinámico' y 'teatral'. En lo que sigue, presentamos la Tabla 3 y el Gráfico 2, donde se expone la frecuencia de evaluaciones registrada en la muestra, incluyendo las variables sexo y estrato social de los informantes.

Según los datos presentados en la Tabla 3 y en el Gráfico 2, aunque son notables las semejanzas en el empleo de este constituyente de las narrativas, dentro de cada estrato hay que identificar algunas diferencias de género. De esta forma, precisamos que la evaluación es uno de los componentes de la estructura narrativa que, en todas las ocasiones, manifiesta un registro mayor por parte de las mujeres en los cuatro estratos sociales. Así, en el estrato medio alto las mujeres registran un $20,4 \%$ (55/268) de las evaluaciones en comparación con los hombres que apenas las emplean en el 7,8\% $(21 / 268)$ de sus narraciones. En el estrato medio, por su parte, se observa el 15,8\% (4I/268) de los casos de evaluaciones en mujeres, a diferencia de los hombres que las emplean en el 6,7\% (18/268) de los casos.

Por su parte, en el estrato medio bajo, las frecuencias de empleo de dicho componente de las narrativas en hombres y en mujeres se acercan considerablemente: II,6\% $(3 \mathrm{I} / 268)$ en el caso de las hablantes femeninas y II,2\% (30/268) en los hablantes masculinos.

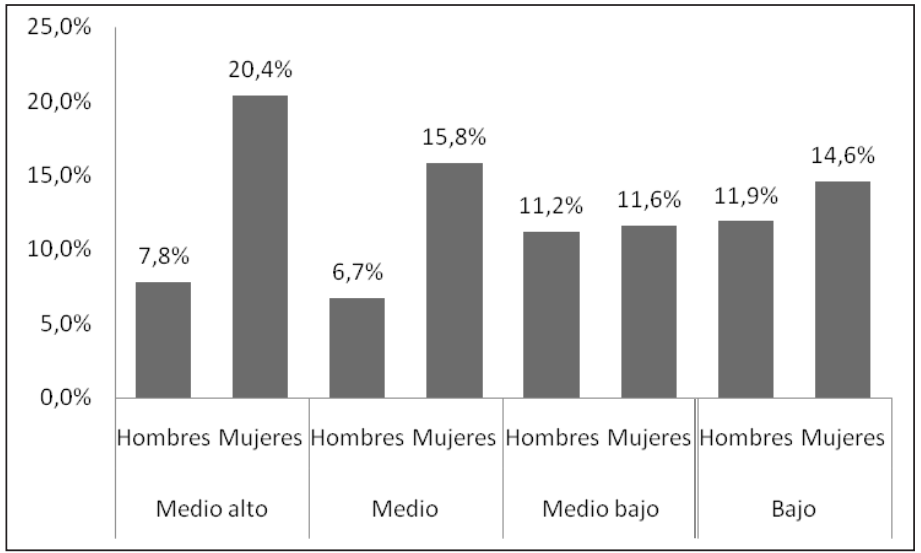

Gráfico 2. Frecuencia de evaluaciones registrada en el corpus. 
Finalmente, en el estrato bajo, las mujeres emplean este recurso en el $14,6 \%(40 / 268)$ a diferencia de los hombres, que registran el II,9\% (32/268). Pese a estos antecedentes, no se observan diferencias de género en la ubicación de la evaluación en los relatos, esto es, tanto en hombres como en mujeres se manifiesta transversalmente, como veremos en los ejemplos de la siguiente sección.

Es necesario señalar, sin embargo, que dentro del componente evaluativo se registran importantes diferencias de género en cuanto a las categorías evaluativas que componen la categoría en cuestión. En el apartado que sigue se analizarán dichas categorías.

\section{I.I. Categorías evaluativas}

La evaluación da cuenta del componente afectivo de las narraciones, es decir, a través de la evaluación se narra algo de la actitud de los hablantes o de la importancia de los hechos acaecidos para quien cuenta la historia. Una de las formas de manifestación de las evaluaciones es por medio de distintas categorías evaluativas, como lo son las emociones, los elementos cognitivos, las percepciones, los estados físicos, las intenciones, las relaciones y los estilos de habla de los personajes.
En lo que sigue, presentamos la Tabla 4 y el Gráfico 3 donde se señalan las frecuencias de empleo de las categorías evaluativas utilizadas por los hablantes de la muestra, cada una de dichas categorías identificada con las siglas propuestas por Jiménez (2006): emoción (EMO), cognición (COG), percepción (PER), estado físico (PHY), intención (INT), relación (REL), habla reportada (RPS), habla reportada directa (RPD), habla reportada indirecta (RPI) y habla reportada libre (RPF).

Como puede observarse en la Tabla 4 en el Gráfico 3 , por lo que respecta a las categorías evaluativas registradas en las evaluaciones de los relatos que conforman nuestro corpus, observamos que, en la mayoría de los casos, son las mujeres las que emplean la mayor cantidad de elementos evaluativos y que, además, se producen algunas diferencias interesantes en la frecuencia de registro de las mencionadas categorías por los informantes de ambos sexos. La mayor diferencia se observa en la utilización de habla reportada directa (RPD), donde las mujeres la emplean en el $18,7 \%(48 / 62)$ de las ocasiones del total de ocurrencias de la muestra, mientras que los hombres solo la usan en el 5,2\% ( $14 / 62$ ) de los casos. Le sigue en cuanto a diferencia de frecuencia el empleo de habla reportada indirecta (RPI), registrándose un uso del II\% (29/39) de

Tabla 4. Tabla general de frecuencia de categorías evaluativas registradas en el corpus.

\begin{tabular}{|l|c|c|c|c|c|c|c|c|c|}
\hline \multirow{2}{*}{ Sexo } & \multicolumn{9}{|c|}{ Frecuencia categorías evaluativas } \\
\cline { 2 - 10 } & EMO & COG & PER & PHY & INT & REL & \multicolumn{3}{|c|}{ RPS } \\
\cline { 5 - 10 } & & & & & & & RPD & RPI & RPF \\
\hline Hombres & 4 & 30 & 27 & 16 & 0 & I & I4 & 10 & 2 \\
\hline Mujeres & 10 & 30 & 38 & 4 & 2 & 0 & 48 & 29 & 3 \\
\hline Totales & 14 & 60 & 65 & 20 & 2 & I & 62 & 39 & 5 \\
\hline
\end{tabular}

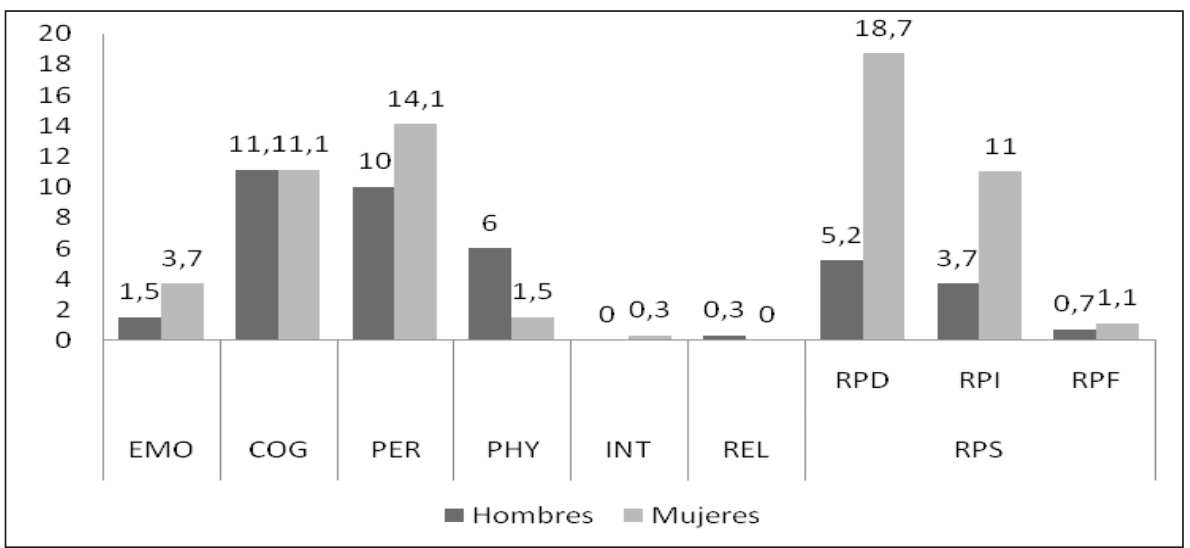

Gráfico 3. Gráfico general de frecuencia de categorías evaluativas registradas en el corpus (\%). 
ocasiones del total de casos de nuestro corpus en los relatos femeninos en comparación a los masculinos, que solo registran el 3,7\% (10/39) de ocurrencias. En tercer lugar, se registra una diferencia significativa en el uso de la percepciones (PER), donde las mujeres las empleanen el I4, I\% (38/65) de los casos, mientras que los hombres hacen uso de este tipo de categoría en el 10\% (27/65) de ocurrencias del total de la muestra. Las emociones (EMO) también se observan con mayor frecuencia en el habla femenina, pues en la muestra se registró el $3,7 \%(10 / 14)$ de los casos en los relatos de las mujeres, en oposición al I,5\% (4/I4) de ocurrencias en los relatos de los hombres. El único caso de una categoría evaluativa que registre mayor frecuencia de empleo por parte de los informantes masculinos de la muestra se observa en los estados físicos (PHY), donde ellos registran el 6\% (16/20) de las ocasiones, mientras que las mujeres incluyen esta categoría en apenas el I,5\% de los casos (4/20). En el caso de las cogniciones (COG), las relaciones (REL), las intenciones (INT) y el habla reportada libre
(RPF), las diferencias son mínimas o, simplemente, no existen. A continuación, presentamos la Tabla 5 y el Gráfico 4, donde se expone la frecuencia de categorías evaluativas registrada en la muestra, incluyendo las variables sexo y clase social.

Como se observa en la Tabla 5 y en el Gráfico 4, el empleo de categorías evaluativas por parte de los informantes, considerando su estrato social, manifiesta diferencias muy leves en cuanto a la frecuencia de empleo registrada en los hombres y las mujeres de la muestra. Sin embargo, consideramos necesario destacar, del mismo modo que en el apartado anterior, algunas diferencias: en lo que respecta a los tipos de habla reportada (RPS), tanto el habla reportada directa (RPD) como la indirecta (RPI), generalmente, registran una frecuencia mayor en las narraciones femeninas de todos los estratos de la muestra. Asimismo, las mujeres recurren, en general, a percepciones (PER) y a emociones (EMO) con mayor facilidad que los hombres para evaluar sus historias. Lo contrario ocurre con los estados físicos

Tabla 5. Frecuencia de categorías evaluativas empleadas en el corpus.

\begin{tabular}{|c|c|c|c|c|c|c|c|c|c|c|}
\hline \multirow{3}{*}{ Estrato } & \multirow{3}{*}{ Sexo } & \multicolumn{9}{|c|}{ Frecuencia categorías evaluativas } \\
\hline & & \multirow[b]{2}{*}{ EMO } & \multirow[b]{2}{*}{ COG } & \multirow[b]{2}{*}{ PER } & \multirow[b]{2}{*}{ PHY } & \multirow[b]{2}{*}{ INT } & \multirow[b]{2}{*}{ REL } & \multicolumn{3}{|c|}{ RPS } \\
\hline & & & & & & & & RPD & RPI & RPF \\
\hline \multirow{2}{*}{$\begin{array}{l}\text { M e d i o } \\
\text { alto }\end{array}$} & Hombres & I & 6 & 5 & 5 & 0 & $\mathrm{I}$ & 1 & 0 & 0 \\
\hline & Mujeres & 2 & 6 & 12 & 1 & $\mathrm{I}$ & 0 & 30 & 3 & $\mathrm{I}$ \\
\hline \multirow[t]{2}{*}{ Medio } & Hombres & $\mathrm{I}$ & 7 & 6 & 3 & 0 & 0 & I & 3 & 0 \\
\hline & Mujeres & 3 & 11 & 9 & 0 & 0 & 0 & 11 & 4 & 0 \\
\hline \multirow{2}{*}{$\begin{array}{l}\text { M e d i o } \\
\text { bajo }\end{array}$} & Hombres & 2 & 7 & 8 & 4 & 0 & 0 & 2 & 5 & 2 \\
\hline & Mujeres & $\mathrm{I}$ & 6 & 7 & 3 & $\mathrm{I}$ & 0 & 4 & 8 & $I$ \\
\hline \multirow[t]{2}{*}{ Bajo } & Hombres & 0 & 10 & 8 & 4 & 0 & 0 & 8 & 2 & 0 \\
\hline & Mujeres & 4 & 7 & 10 & 0 & 0 & 0 & 3 & 14 & $I$ \\
\hline
\end{tabular}

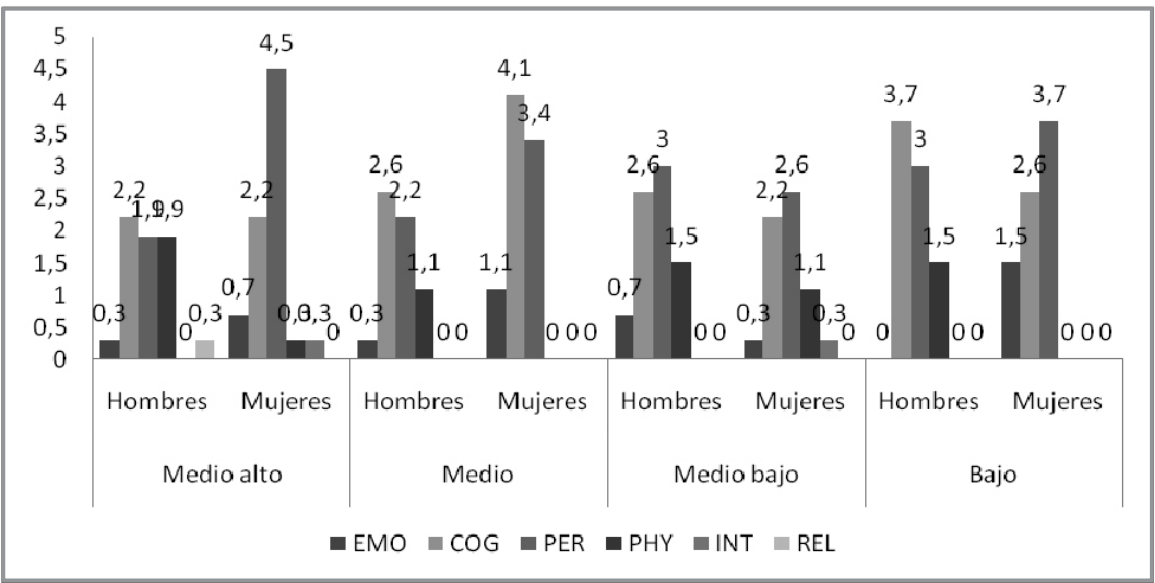

Gráfico 4. Frecuencia de categorías evaluativas registradas en el corpus (\%). 
(PHY), pues en los cuatro estratos analizados son los hombres los que emplean con mayor frecuencia las descripciones físicas como elemento evaluativo en sus narraciones. El resto de las categorías, como señalamos anteriormente, presenta frecuencias de empleo muy leves tanto en los hombres como en las mujeres de los estratos sociales en estudio.

Respecto de las evaluaciones y las categorías evaluativas, exponemos a continuación los ejemplos más representativos de dichos rasgos, no sin antes aclarar que para facilitar la comprensión de los fenómenos analizados en esta sección se utilizarán las siguientes convenciones en la presentación de los ejemplos:

- Se transcribirá cada ejemplo en forma de cita incluyendo la cantidad de contexto necesaria para su comprensión 6 .

- En cada cita se marcará con negrita el segmento en el cual se expresa la categoría evaluativa correspondiente. Luego, se identificará cada una de dichas categorías con las siglas propuestas por Jiménez (2006).

- Al final de cada cita se pondrá, entre paréntesis, el código del sujeto que la emitió.

(I) I: eeeh a bueno ¿en el colegio por ejemplo decíh tú? eh $\mathrm{mmm}$ sí poh/ igual nosotroh éramoh súper desordenadoh (PER) mi curso éramoh super unidoh y desordenadoh (PER) me acuerdo que teniamoh un botiquín/ y eeh obviamente uno en loh colegioh loh botiquineh no se pone no se echa na[da] (PER) lo botiquineh to[d]o vacío y lo ocupábamoh pa[ra] dihtinta cosa/ por ejemplo/ al principio era la cabina telefónica/ un compañero trajo el teléfono del hermano chico y lo puso ahí en el en el botiquín poh/ entonceh eh por ejemplo ehtábamos en claseh y to[d]o empezábamoh ¡teléfono! ¡teléfono! (RPS) y la profesora tenía que pararse y contehtar el teléfono y nosostroh la, otro compañero le contehtaba cuetioneh/ despuéh era fax/ porque fuimoh progresando (PER)/ y también mih compañeroh leh ponían papeleh con siempre con la minma profesora la profe de hihtoria y como que noh aguantaba to[d]a la cuehtioneh que hacíamoh (PER) y le ponían le ponían aparte que era bonita (PER) ella entonceh le ponían en el fax mih compañero le ponían por ejemplo profe hoy día se ve linda con el pañuelo rojo(RPS)/ mi entonceh empezábamoh fax fax fax fax (RPS)/ y la profe se tenía que parar a contehtar a ver qué papel había poh y lo leía y le pedíamoh que lo leyera en voh alta/ y se ponía roja de vergüenza (PHY) y un día un día de invierno hacía mucho frío en el colegio en la sala/ y una compañera le dice a otro compañero/ oye sabíh que tengo frío (RPS)/ eh ya no te preocupí yo te voy a ayudar (RPS) y mi compañero no encontró nada mejor (PER) de que agarrar papel lo mete dentro del botiquín y le prende fuego (INT) a la cuestión empezó a salir humo llegó el inhpector indignado (PER) y ahí noh y ahí noh sacaron el botiquín poh/ lo tuvieron que sacar porque ya era mucho el ehcándalo (PER) con el famoso botiquín (M075, estrato medio bajo).

(2) I: tenía// tuvimoh una profe de primero a cuarto básico / como la típica profe que inicia a loh niñoh (PER) // y era muy mala (PER) noh trataba pero muy mal así como maltrato sicológico brígido (PER) y yo era súper floja (PER) / todo el colegio / o sea malah notah y como desordenada (PER)/ desordenada sobre to[d]o (PER) y estábamoh como con mih amigah / la Simone / la Naty / como ¿qué vai a ser cuando grande? (RPS) / recreo / un día / segundo básico / y yo eeh onda como yo quiero ser actrih, yo quiero ser no sé qué (RPS) no me acuerdo poh / y yo dije como yo quiero ehtudiar medicina (RPS) porque mi hermano mayor quería ehtudiar medicina en ese tiempo/ y yo en realida[d] ni siquiera conocía lah cosah / no sé por qué quería eso / medicina (COG) / y pasó la tía Tamara que me odiaba (PER) y me dijO / pero con lah notah que tenla no te alcanza ni pa[ra] ehtud ni $\mathrm{pa}$ [ra] ser ascensorhita (RPS) /cacha la vieja mala poh (COG) y yo le dije eeh / me sentí tan triste (EMO) y le dije como obliga[d]a a ser profesora básica (RPS) a y me agarró como de un brazo / me llevó a in[s]pectoría (INT) y quedé condicional en segundo básico porque la tía Tamara dijo como que yo le había falta[d] o mucho el respeto (RPS) condicional / eso es loh mah... (PER) que me ha pasa[d]o en el colegio parece (MI97, estrato medio alto).

(3) I: eh/ una bueno/ de lah cosah simpáticah que me acuerdo (PER) yo que me pasó en el colegio fue cuando iba en octavo básico/ en el año noventa y cinco y noh hicieron un paseo al museo bellah arteh/ entonceh/ típico cabro chico en loh paseoh al bellah arteh se pone a tontear con loh amigoh ¿cierto? (COG)/ y me acuerdo yo que el pasillo em algunoh cuadroh y to[d]o / el piso era rehbaloso/ y to[d]oh/ corrían y se rehbalaban poh/ tu cachai/ y yo el simpático (PER) se me ocurrió hacer lo mihmo con mih compañeroh/ y juhto yo me rehbalo y llego 
a una parte que ehtá enserá[da]/ y se acabó la parte de reh de rehbalín/ entonceh yo iba corriendo/ me empiezo a rehbalar/ llego a esa parte/ y se me frenan loh pieh y caigo de rodillah y me hice como/ como una ehtrella de rock/ pero me saqué crehta y media/ y lo mah gracioso (PER) eh que yo me paro y to[d]o el había mucha gente en el museo/ entonceh ehtaban loh ehtudianteh de arte de arquitectura y cuehtioneh/ que van a ver/ y ehtaban to[d]oh mirándome con cara de que ehte pelotu[d]o que ehtaba haciendo ahí (COG) y yo me paro corriendo/ mih compañeroh se habían arranca[d] o y me paro corriendo gritándoleh ¡chiquilloh ehpérenme! (RPS) y la cuehtión/ así que fue el manso show (PER) fue una experiencia bien chihtosa (PER) (HI22, estrato medio).

(4) I: me acuerdo que cuando no sél en el liceo cuando llegaban/ compañeroh gordoh (PHY) atrasa[d]oh/ alguna cosa así/ como generalmente loh dejaban afuera y leh cerraban la puerta no loh dejaban entrar/ ehh/ se sentaban to[d]oh y lo dejaban entrar poh/ [en]tonceh cuando llegaba y se sentaba saltaba toda la sala/ [en]tonceh el hueon se ponía rojo (PHY) como que entraba él/ como que no lo/ le/ loh tiraban pa[ra] [e]l patio/ loh tiraban $\mathrm{pa}[\mathrm{ra}]$ fuera de la sala/ afirmaban la puerta y cuando entraba y se sentaba saltaban todoh/ y lo hicieron una veh con una profe y la profe se enojó.

E: a una profe se lo hicieron

I: sí

E: ila profe era gorda?

I: un poquito entra[d]ita en carne (PHY)

(HI26, estrato medio).

En(I),lainformantematizasurelato,fundamentalmente, con percepciones ("éramoh súper desordenadoh", "éramoh súper unidoh y desordenadoh", "porque fuimos progresando", "aparte que era bonita”, “y era mucho el ehcándalo", etc.) y habla reportada, sobre todo, indirecta ("y todosh empezábamoh jteléfono! ¡teléfono!", "entonceh empezábamoh fax fax fax", "oye tengo frío/ ya no te preocupih, yo te voy a ayudar", etc.). Además, en esta narración se registra una de las únicas intenciones de la muestra ("mi compañero no encontró nada mejor de que agarrar papel lo mete dentro del botiquín y le prende fuego"), producida por una representante de los relatos femeninos.

En (2), por su parte, la situación es bastante similar a la anterior, pues, una vez más, nuestra narradora incluye percepciones ("como la típica profe que inicia a loh niñoh", "era muy mala", "noh trataba pero muy mal, maltrato sicológico brígido", "y yo era súper floja”, "desordenada sobre todo", etc.) y habla reportada ("iqué vai a ser cuando grande?", "y yo eeh onda como yo quiero ser actrih", "y yo dije como yo quiero ehtudiar medicina", etc.) en su historia.Asimismo, incorpora emociones ("me sentí tan triste") y estados cognitivos ("cacha la vieja mala poh"), que serán la tónica de las historias de las mujeres de la muestra analizada.

En (3), por otro lado, observamos que el entrevistado inicia y cierra su discurso con percepciones ("cosah simpáticah que me acuerdo", "fue una experiencia bien chihtosa"), además de incluir estados cognitivos ("típico cabro chico") y un caso de habla reportada ("chiquilloh, espérenme"). Si bien los hablantes de ambos sexos, en ciertas ocasiones, abren y finalizan sus relatos con la misma percepción, la mayor frecuencia de estos casos se observa en las narraciones masculinas. Asimismo, como se aprecia en (4), una de las estrategias recurrentes de los relatos masculinos fue incluir más frecuentemente estados físicos de los personajes en los relatos (“compañeroh gordoh”,"el weón se ponía rojo”,"un poquito entradita en carne").

En los ejemplos citados anteriormente, podemos observar que, si bien, hombres y mujeres emiten evaluaciones de manera transversal en sus relatos, son las mujeres las que, en general, construyen narraciones con más recursos evaluativos, especialmente, cuando se trata de elementos dialógicos. Por consiguiente, en las narrativas femeninas se incorpora una mayor frecuencia tanto de estilo directo como de indirecto.

Respecto de la importancia del estilo directo en las narraciones, debemos destacar que nuestros hallazgos coinciden con los de Prieto y San Martín (2002-2003), pues es este tipo de discurso el que dota de un potencial dinamizador y expresivo a las historias elaboradas por mujeres. Asimismo, conviene precisar que son las informantes femeninas las que incluyen más percepciones y emociones como recursos evaluativos en sus relatos, mientras que los hombres se destacan por no incorporar gran variedad de estrategias evaluativas; aunque a diferencia de ellas, suelen incluir aspectos físicos, siendo más concretos en sus narraciones, por lo tanto, que las mujeres. 


\section{CONCLUSIONES}

A partir del análisis realizado a las 64 narraciones (32 femeninas y 32 masculinas) que conforman la muestra de nuestro estudio, concluimos que el componente evaluativo no mostró grandes diferencias de género en cuanto a su frecuencia de aparición en los relatos, pues se registró en 61/64 ocasiones y, de estas, 29/6I pertenecen a hombres y $32 / 6$ I a mujeres. El componente en estudio registró 268 ocurrencias distribuidas en las 6I narraciones mencionadas anteriormente, de las cuales 167/268 $(62,3 \%)$ casos corresponden a mujeres y $101 / 268$ $(37,7 \%)$ a hombres. Asimismo, para este recurso, si consideramos la variable estrato social, se registran algunas diferencias de género significativas, pues en los cuatro estratos en estudio las mujeres superan a los hombres en la utilización de evaluaciones, observándose las mayores frecuencias en las mujeres de los estratos medio alto $(20,4 \%$ de casos en las mujeres en comparación a 7,8\% en los hombres) y medio (I5,8\% para las mujeres y $6,7 \%$ para los hombres) seguidas de las mujeres de los estratos bajo ( $11,9 \%$ en el caso de ellas y $11,9 \%$ en los hablantes masculinos) y medio bajo (II,6\% de ocurrencias registradas en las mujeres versus los hombres que las emplean en I I,9\% de las ocasiones). En este sentido, el análisis del componente evaluativo resultó altamente productivo. Pese a estos datos, no se registraron diferencias de género en la ubicación de la evaluación en los relatos, es decir, tantos en hombres como en mujeres se manifiesta transversalmente.

Por otra parte, dentro del componente evaluativo se registran importantes diferencias de género en cuanto a las categorías evaluativas que lo conforman. De esta forma, podemos señalar que son las mujeres las que utilizan la mayor cantidad de elementos de evaluación, sobre todo, en cuanto a la utilización de estilo directo, indirecto, percepciones y emociones, coincidiendo con los hallazgos de Soler (2004) en su estudio sobre las diferencias de género registradas en los relatos de hombres y mujeres en Bogotá. Dicha autora concluye que es en la evaluación donde se observa una diferencia de género fundamental, esto es, que las mujeres emplean casi el doble de elementos evaluativos que los hombres. En el caso de los informantes masculinos, destaca, de manera especial, el alto empleo de estados físicos como estrategia evaluativa, transformándose, de este modo, en el único recurso que registró índices de frecuencia superiores a los de las informantes mujeres que conformaron la muestra de nuestro trabajo. De esta forma, se podría vincular a las mujeres con los aspectos afectivos y la comunicación de sentimientos en la historia, mientras que a los hombres con los aspectos más concretos, donde lo más importante son las acciones y la comunicación de información por sobre la justificación de la historia, dada por el componente evaluativo (Lakoff 1973; Tannen 1990a, 1990b, 1996; Soler, 2004; Coates, 2009).

Por último, podría interpretarse la mayor frecuencia del componente evaluativo en el discurso narrativo femenino como un producto de la división establecida a principios del siglo XIX, entre el dominio público y el privado, que instala, de acuerdo con Hall (1995), a los hombres en la esfera de los negocios, del comercio y de la política, y a las mujeres en el mundo privado de la familia y el hogar, donde más que el intercambio de información, importa el establecimiento y mantenimiento de las relaciones sociales.

En este sentido, podría vincularse el hecho de que las mujeres relaten sus historias de un modo mucho más real, más lógico y más 'entretenido' que los hombres -validándolas, constantemente, a través de elementos evaluativos-, debido a su cercanía con las prácticas discursivas de la oralidad, sobre todo, y coincidiendo con Prieto y San Martín (20022003), en la relativa mayor utilización del discurso referido. Son las mujeres las que, al parecer, tienen una mayor conciencia de la importancia de la cultura oral y al emplearla más, la desarrollan mejor, incluyendo, según la propuesta de estos autores, pautas discursivas que parecieran vincularse con la identidad de género. 


\section{REFERENCIAS BIBLIOGRÁFICAS}

Adam, J. (1999). Linguistique textuelle. Des genres de discours aux textes. Paris: Nathan/HER.

Bassols, M. \& Torrent, A. (2003). Modelos textuales. Barcelona: Eumo-Octaedro.

Blas Arroyo, J. L. (2005). Sociolingüística del español. Desarrollos y perspectivas en el estudio de la lengua española en contexto social. Madrid: Cátedra.

Calsamiglia, H. \& Tusón, A. (1999). Las cosas del decir. Manual de análisis del discurso. Barcelona: Ariel.

Charaudeau, P. \& Maingueneau, D. (2005). Diccionario de análisis del discurso. Buenos Aires: Amorrortu editores.

Cheshire, J. (2003). Sex and gender in variationist research. En J. Chambers, P. Trudgill \& N. Schilling-Estes (Eds.), The handbook of language variation and change (pp. 423-443). Oxford: Blackwell Publishing.

Coates, J. (2009). Mujeres, hombres y lenguaje. Un acercamiento sociolingüístico a las diferencias de género. Fondo de Cultura Económica: México, D. F.

De Beaugrande, R. (2000). La saga del análisis del discurso. En T. van Dijk (Comp.), El discurso como estructura y proceso (pp. 67-106). Barcelona: Gedisa.

Hall, K. (1995). Lip service on the fantasy lines. En K. Hall \& M. Bucholtz (Eds.), Gender articulated: Language and the constructed self (pp. I83-216). Nueva Cork/Londres: Routledge.

Jiménez, T. (2006). La narración infantil. Un estudio en niños de educación básica. Revista de investigación, 60, I57-174.

Jones, D. (1980). Gossip: Notes on women's oral culture. En C. Kramarae (Ed.), The voices and words of women and men (pp. 193-198). Oxford: Pergamon Press.

Labov, W. (1972). The transformation of experience in narrative syntax. Language in the inner city. Studies in the Black English Vernacular. Filadelfia: University of Pennsylvania Press.

Labov,W. (1992). Modelos sociolingüísticos. Madrid: Cátedra.

Labov, W. \& Waletzky, J. (1967). Narrative analysis. En J. Helm (Ed.), Essays on the verbal and visual arts (PP. 12-44). Seattle: University of Washington Press.

Lakoff, R. (1973). Language and woman's place. Language in Society, 2, 45-79.

López Morales, H. (2004). Sociolingüística. Madrid: Gredos.

Moreno Fernández, F. (1998). Principios de sociolingüistica y sociología del lenguaje. Barcelona: Ariel.

Prieto, L. (1995-1996).Análisis sociolingüístico del dequeísmo en el habla de Santiago de Chile. Boletín de Filología, 379-452.

Prieto, L. \& San Martín, A. (2002-2003). Diferencias de género en el empleo del discurso referido:Aproximación sociolingüística y pragmático-discursiva. Boletín de Filología, 269304.

Shiro, M. (1998). Los pequeños cuentacuentos. El desarrollo de las habilidades narrativas en niños de edad escolar. Caracas: Universidad Central de Venezuela.

Soler, S. (2004). Discurso y género en historias de vida. Una investigación de relatos de hombres y mujeres en Bogotá. Publicaciones del Instituto Caro y Cuervo: Bogotá.

Tannen, D. (1990a). La comunicación entre hombres y mujeres a la hora del trabajo. Buenos Aires: Javier Vergara Editor S.A. 
Tannen, D. (I990b). You just don't understand. Women and man in conversation. Nueva York: Ballantine Books.

Tannen, D. (1996). Género y discurso. Barcelona: Paidós.

van Dijk, T. (2000). Superestructuras. La ciencia del texto. Barcelona/Buenos Aires: Paidós.

van Dijk, T. (2005). Estructuras y funciones del discurso. Una introducción interdisciplinaria a la lingüística del texto y a los estudios del discurso. Madrid: Siglo Veintiuno Editores.

Werlich, E. (1975). Typologie der Texte. Munich: Fink.

\section{NOTAS}

I.- Según estos autores, la oralidad primaria no se condice con un retraso en la marcha hacia la racionalidad y la modernidad, porque en el ámbito sociológico, el paso a la modernidad ha sido descrito empleando la conceptualización del sociólogo alemán Ferdinand Tönnies, como un tránsito de Gemeinschaft -que define el tipo de relaciones sociales entre individuos con vínculos interpersonales estrechos e íntimos, preocupación por el bienestar de los demás y cooperación y confianza mutuas, además del valor asignado a los derechos y obligaciones tradicionales -a la Gesellschaft- que define un tipo de sociedad en la que las relaciones sociales se caracterizan por la competencia, el interés propio, la eficiencia, el progreso y la especialización, además del desapego de los lazos tradicionales.

2.- De acuerdo con Labov (1992), el objetivo de la investigación lingüística de una comunidad de habla consiste en estudiar la forma en cómo habla la gente cuando no está siendo sistemáticamente observada; sin embargo, solo podemos obtener tales datos mediante la observación sistemática. $A$ esto es a lo que se le ha denominado 'paradoja del observador'. Este dilema se soluciona encontrando la manera de completar la entrevista formal con otros datos, o cambiar la estructura de la situación de la entrevista de una u otra manera. En este sentido, una forma de superar la paradoja consiste en romper las constricciones de la situación de entrevista mediante diversos procedimientos que distraen la atención del sujeto en su propio discurso y pueden hacer brotar su habla más vernácula.

3.- El equipo PRESEEA, Santiago de Chile, está compuesto por los profesores de la Universidad de Chile: Alfredo Matus, Luis Prieto (coordinadores), Abelardo San Martín y Silvana Guerrero (asistentes). El proyecto ESECH también corresponde a un grupo de estudio de la Universidad de Chile.
4.- El código de los sujetos tiene las siguientes equivalencias: sexo: $\mathrm{H}$ (hombre) y $\mathrm{M}$ (mujer). El número que le sigue a dichas equivalencias corresponde a la numeración correlativa que se le asignó a los informantes en el corpus del ESECH.

5.- Respecto de la transcripción de los ejemplos, es preciso indicar las siguientes convenciones: I) $\mathrm{E}=$ entrevistador e I= informante; 2) el texto correspondiente a cada entrevista ha de transcribirse en ortografía convencional, incluidos los acentos gráficos. Las palabras que presentan elisiones en su pronunciación se completan en su escritura con paréntesis cuadrados en el caso de las aspiraciones, estas se marcarán con h; 3) para la mejor comprensión de los ejemplos que hemos incluido en este artículo, se ha suprimido el sistema de de etiquetas pertenecientes al Standard Generalized Markup Language (SGML) contenidas en el Text Encoding Initiative (TEI), que se emplean en la transcripción de las entrevistas pertenecientes al PRESEEA, excepto las pausas.

6.- Respecto de la transcripción de los ejemplos, es preciso indicar las siguientes convenciones: I) $\mathrm{E}=$ entrevistador e I= informante; 2) el texto correspondiente a cada entrevista ha de transcribirse en ortografía convencional, incluidos los acentos gráficos. Las palabras que presentan elisiones en su pronunciación se completan en su escritura con paréntesis cuadrados en el caso de las aspiraciones, estas se marcarán con h; 3) para la mejor comprensión de los ejemplos que hemos incluido en este artículo, se ha suprimido el sistema de etiquetas pertenecientes al Standard Generalized Markup Language (SGML) contenidas en el Text Encoding Initiative (TEI), que se emplean en la transcripción de las entrevistas pertenecientes al PRESEEA, excepto las pausas. 